\title{
microRNAs mediate oleic acid-induced acute lung injury in rats using an alternative injury mechanism
}

\author{
SANG MOOK LEE ${ }^{1},{\text { HYUNSOO } \mathrm{CHOI}^{2} \text {, GEUMJIN YANG }}^{2}$, KI CHEOL PARK ${ }^{2}$, \\ SIKYOUNG JEONG ${ }^{3}$ and SUNGYOUP $\mathrm{HONG}^{3}$ \\ ${ }^{1}$ Department of Anaesthesia and Pain Medicine; ${ }^{2}$ Clinical Research Institute; ${ }^{3}$ Department of Emergency Medicine, \\ Daejeon St. Mary's Hospital, College of Medicine, The Catholic University of Korea, \\ Seocho-gu, Seoul 137-701, Republic of Korea
}

Received September 9, 2013; Accepted March 17, 2014

DOI: $10.3892 / \mathrm{mmr} .2014 .2155$

\begin{abstract}
Intravenous (IV) infusion of oleic acid (OA) distributes OA microemboli in the pulmonary capillaries, which results in severe vascular congestion, hemorrhage vascular congestion, interstitial edema, intravascular coagulation and bleeding. The immune response to acute lung injury (ALI) is known to be associated with rapid and widespread changes in microRNA (miRNA) expression in the lung. The present study of a model of rat lung injury aimed to investigate how the lung miRNA profile changes to mediate ALI. For the induction of ALI, OA $(200 \mu \mathrm{l} / \mathrm{kg})$ suspended in $20 \%$ ethyl alcohol was injected through the tail vein for $20 \mathrm{~min}$. Lung tissue samples were acquired at 3,6 and $24 \mathrm{~h}$, and miRNA microarray and quantitative polymerase chain reaction were performed using these samples. The activation of phosphatase and tensin homolog (PTEN), protein kinase $\mathrm{B}(\mathrm{Akt})$, extracellular signal-regulated kinases (ERK) and c-Jun N-terminal kinases (JNK) were analyzed by western blot analysis. There were 75 miRNAs that demonstrated $>1.5$-fold changes in expression levels. miR-101a was highly upregulated at $3 \mathrm{~h}$. miR-21 was upregulated in the OA group throughout the $24 \mathrm{~h}$ following OA challenge. miR-1 was the most downregulated miRNA at $24 \mathrm{~h}$. In order to examine the expression levels of PTEN and Akt as targets of miR-21, western blot analysis was performed. At $3 \mathrm{~h}$, the levels of PTEN were attenuated in the OA group as compared with those in the control group; however, p-Akt/Akt levels were increased at $3 \mathrm{~h}$ for the OA group. PTEN and p-Akt/Akt were significantly higher in the OA group at $3 \mathrm{~h}$ and were rapidly decreased at $6 \mathrm{~h}$. The immunohistochemical stain of $\alpha$-smooth muscle actin in the bronchial and alveolar wall increased $24 \mathrm{~h}$ after OA-induced ALI. These results indicated
\end{abstract}

Correspondence to: Professor Sungyoup Hong, Department of Emergency Medicine, Daejeon St. Mary's Hospital, 64 Daeheungro, Junggu, Daejeon 302723, Republic of Korea

E-mail: emhong@gmail.com

Key words: microRNAs, acute lung injury, oleic acid, intracellular signaling proteins that the profile of miRNAs dynamically changed throughout the OA-induced ALI process, and mitogen-activated protein kinase activation, PTEN/Akt pathway alteration and smooth muscle actin activation were observed in this ALI model.

\section{Introduction}

Pulmonary fat embolism is a common etiology of morbid lung disease that occurs with long bone fracture (1). Intravenous (IV) infusion of oleic acid (OA) has been widely studied as an acute lung injury (ALI)/acute respiratory distress syndrome (ARDS) model and mimics the pathological process clinically observed in pulmonary embolism, pancreatitis and meconium aspiration (2). The small OA microemboli in pulmonary capillaries results in severe vascular congestion, interstitial edema, intravascular coagulation and alveolar hemorrhage $(3,4)$. Despite the potential applications as a model for ALI, the mechanism of OA-induced ALI is far from fully understood.

microRNAs (miRNAs) are noncoding RNAs that have been issued as important regulators of gene expression. miRNAs are important in the regulation of gene expression by RNA interference (RNAi), which involves endogenously induced gene silencing achieved by binding to specific target messenger RNA (mRNA) sequences at the posttranscriptional level resulting in null or hypomorphic phenotypes (5). A diverse range of miRNAs have been reported as critical components in a comprehensive model of inflammatory lung disease with roles in the regulation of immune and inflammatory responses, and these miRNAs are novel therapeutic targets and biomarkers for ALI/ARDS (6). The immune response to aerosolized lipopolysaccharide (LPS) is associated with widespread, rapid increases in miRNA expression in the mouse lung (7). Additionally, an expression profiling of miRNAs has been analyzed in bleomycin-induced lung injury and in lung fibrosis, and the potential target genes of miRNAs may contribute to complex transcriptional processes, including apoptosis, toll-like receptor (TLR) signaling and transforming growth factor- $\beta$ signaling pathways (8).

The first purpose of the present study was to determine the expression pattern of miRNAs in OA-induced ALI. 
The second purpose was to investigate how the expressed miRNAs mediate ALI.

\section{Materials and methods}

Animal preparation and induction of ALI. Male Sprague-Dawley rats with a body weight of 200-220 g were obtained from Samtako (Osan, Gyeonggi, Korea) and were kept for 1 week at $23 \pm 2^{\circ} \mathrm{C}$ with a regular day/night cycle with access to tap water ad libitum. All procedures were approved by the Institutional Animal Care and Use Committee at the Catholic University of Korea (Seoul, Korea).

All animals were anesthetized by intraperitoneal injection of zoletil (50-80 mg/kg)/xylazine (5-10 mg/kg). According to the group assignment, the rats were injected with chemical reagents through the lateral tail vein. The OA group was challenged with OA $(200 \mu \mathrm{l} / \mathrm{kg})$ suspended in $20 \%$ ethyl alcohol for $20 \mathrm{~min}$. The control group was challenged with the same volume of $20 \%$ ethyl alcohol. The animals were intubated following anesthesia and sacrificed in a chamber filled with $50 \%$ carbon dioxide at 3,6 and 24 h post-challenge.

Sample preparation. Midline sternotomy was performed following sacrification and $10 \mathrm{ml}$ of saline was perfused through the right ventricle of the heart under direct visualization to remove blood from the lungs. The left main bronchus was clamped with a mosquito clamp and bronchoalveolar lavage (BAL) was performed for the right lung by flushing $2 \mathrm{ml}$ of saline five times through the patent right main bronchus. The left lung was harvested and stabilized in $3 \mathrm{ml}$ of RNAlater (Ambion Europe, Huntingdon, UK), stored at $4^{\circ} \mathrm{C}$ overnight and then kept at $-70^{\circ} \mathrm{C}$ until RNA extraction and western blot analysis.

miRNA isolation labeling and microarray analysis. miRNA was isolated using a mirVana miRNA isolation kit (Ambion, Austin, TX, USA), according to the manufacturer's instructions. RNA quality was assessed using the Agilent Bioanalyzer (Agilent Technologies, Santa Clara, CA, USA) and only samples with RNA integrity numbers $>7$ were used for further analysis.

Two lung tissue samples for each group and at each time-point (3, $6 \mathrm{~h}$ and $24 \mathrm{~h}$ after ALI challenge) were used for microarray analysis. Microarray profiling was performed using a GeneChip 2.0 miRNA array (Affymetrix, Santa Clara, CA, USA). The microarray intensity data were analyzed using the significance analysis of microarray test.

Quantitative polymerase chain reaction $(q P C R)$. To validate the microarray data, several upregulated or downregulated miRNAs with fold changes $>2.5$ were selected and analyzed by qPCR. qPCR ( $n=4$ per group) was performed using specific primers for the miRNA of interest, followed by qPCR with TaqMan MicroRNA assay primers (TaqMan MicroRNA Assay Reverse Transcription Primers; Applied Biosystems, Foster City, CA, USA). The expression levels of each mature miRNA were evaluated using the comparative threshold cycle $\left(\mathrm{C}_{\mathrm{T}}\right)$ method, normalized to those of the internal control miR-202 for each condition $\left(\Delta \Delta \mathrm{C}_{\mathrm{T}}\right) . \Delta \Delta \mathrm{C}_{\mathrm{T}}$ of each miRNA between two groups was calculated and compared using the Mann-Whitney U test.
miRNA target prediction and bioinformatics analysis. MiRDB (9) and TargetScan (10) were used to predict target genes for all differentially expressed miRNAs.

To identify the functions of predicted target genes, the functional annotation tool available through Database for Annotation, Visualization and Integrated Discovery (DAVID; http://david.abcc.ncifcrf.gov) was employed. DAVID bioinformatics resource systematically lists genes with enriched (overrepresented) biological annotation (ontology terms) relative to all annotated genes associated and then statistically highlights the most overrepresented biological annotation. Annotation clusters with an enrichment score $>2$ were selected to analyze whether the target genes were the most highly enriched. Subsequently, this was used for functional annotation clustering.

Western blot analysis. Lung tissue lysates were prepared in lysis buffer (150 mM NaCl, $50 \mathrm{mM}$ Tris-HCl; $\mathrm{pH} 7.5 ; 0.1 \%$ SDS, $1 \%$ Triton $\mathrm{X}-100,1 \%$ sodium deoxycholate, $2 \mathrm{mM}$ EDTA; pH 8.0) and freshly added protease inhibitor cocktail tablets (Roche Diagnostics GmbH, Mannheim, Germany). Equal amounts of lysate (50 $\mu \mathrm{g}$ per lane) from each sample were purified by $10 \%$ SDS-PAGE and transferred onto nitrocellulose membranes (Millipore Corporation, Billerica, MA, USA). The membranes were incubated with primary antibodies, which consisted of rabbit polyclonal anti-protein kinase B (Akt), anti-phospho-Akt Ser473 (pAkt), anti-extracellular signal regulated kinase (ERK), anti c-Jun $\mathrm{N}$-terminal kinase (JNK), anti-p-JNK and rabbit monoclonal anti-p ERK-(Thr202/Tyr204), anti-phosphatase and tensin homolog (PTEN; Cell Signaling Technology, Beverly, MA, USA), rabbit monoclonal anti- $\beta$-actin (MP Biomedicals, Solon, OH, USA), anti-ERK, anti-p-ERK (1:1,000; Cell Signaling Technology), anti-JNK and anti-p-JNK (1:1,000; Cell Signaling Technology), rabbit anti-ERK, anti-p-ERK (1:1,000; Cell Signaling Technology), rabbit anti-JNK and anti-p-JNK (1:1,000; Cell Signaling Technology). Subsequently, the membranes were extensively washed with Tris-buffered saline and Tween-20 and re-incubated for $1 \mathrm{~h}$ at room temperature, with horseradish peroxidase-conjugated anti-rabbit immunoglobulin G (Invitrogen Life Technologies, Carlsbad, CA, USA). Quantitative bands were detected and analyzed by chemiluminescence technology using the ChemiDoc $^{\mathrm{TM}}$ XRS+ from Bio-Rad Laboratories (Hercules, CA, USA).

Immunohistochemistry of $\alpha$-smooth muscle actin ( $\alpha$-SMA). An immunohistochemical stain for $\alpha$-SMA in lung tissue was performed on $5 \mu \mathrm{m}$ slides from formalin fixed, paraffin-embedded lung tissue. The slide preparations were washed in phosphate-buffered saline (PBS) for $5 \mathrm{~min}$ and incubated in $0.3 \% \mathrm{H}_{2} \mathrm{O}_{2}$ in $100 \%$ methanol for $30 \mathrm{~min}$ to quench endogenous peroxidase activity. This step was followed by three washes with PBS. Following inhibition for $1 \mathrm{~h}$ in normal serum of the species from which the secondary antibody was raised, the slides were incubated with the primary antibody using a 1:100 working dilution (rabbit polyclonal anti- $\alpha$-SMA antibody; Abcam, Cambridge, MA, USA). The slides were incubated for $1 \mathrm{~h}$ in a humidified chamber at room temperature, followed by three washes 
in PBS. The slides were then incubated under the same conditions with the secondary biotinylated antibody for $1 \mathrm{~h}$ (avidin-biotin complex, Vectastain Elite kit; Vector Laboratories, Inc., Burlingame, CA, USA).

ELISA for interleukin (IL)-13. The measurement of the concentration of IL-13 in bronchoalveolar lavage (BAL) supernatant was performed by ELISA with rat monoclonal primary antibodies and goat polyclonal secondary antibodies, according to the manufacturer's instructions (Cusabio Biotech Co., Ltd., Wuhan, Hubei, China; Cat no. 84290).

Statistical analysis. The differences in the expression ratio of each miRNA, the intensities of the bands from western blot analysis and IL-13 concentrations in BAL supernatant were compared for each time-point between the two groups. The unpaired t-test was used for parametric variables and the Mann-Whitney U test was used in nonparametric variables. Data analysis was conducted using the $\mathrm{R}$ statistical package version 2.15.0 (The R Foundation for Statistical Computing, Vienna, Austria). $\mathrm{P}<0.05$ was considered to indicate a statistically significant difference. The means and standard errors were indicated for each value.

\section{Results}

Development of OA-induced ALI model. To establish a more stable dosage suspension of OA to inject, $50 \%$ ethyl alcohol was used as the suspension vehicle. OA was then administered intravenously and the extent of ALI at different doses in rats was determined to optimize the OA-induced ALI model. The optimal dose of OA was determined as $200 \mu \mathrm{l} / \mathrm{kg}$ with $\sim 20 \%$ mortality.

miRNA microarray screening. To examine the overview of miRNA expression during OA-induced ALI, miRNA microarrays were performed with total miRNA isolated from rat lung tissues harvested at 3,6 and $24 \mathrm{~h}$ after OA challenge. There were 75 miRNAs with expression level changes at more than one point with $>1.5$-fold change in either direction. The selected miRNAs showed fold changes $>1.5$ between the two groups with increasing time (Table I).

In total, 30 miRNAs demonstrated significant differences between the two groups at $3 \mathrm{~h}$ with four of them downregulated. In addition, 10 miRNAs showed statistically significant differences between the two groups. The expression ratios of 29 miRNAs changed by $>1.5$ fold at $6 \mathrm{~h}$, eight of these miRNAs were downregulated and 10 miRNAs demonstrated statistically significant changes in the OA group. In total, 65 miRNAs demonstrated expression changes at $24 \mathrm{~h}$, of which 30 were upregulated with 19 demonstrating statistically significant changes.

$q P C R$. qPCR was performed to validate the microarray data and to identify predictable targets responsible for the development of OA-induced ALI. miRNAs were selected from microarray analysis for expression changes by more than two-fold with statistically significant differences between the two groups. These miRNAs were compared using qPCR. A progressive change in expression levels from
3 to $24 \mathrm{~h}$ was observed in several selected miRNAs. The expression of miR-101a increased by 2.4 -fold at $3 \mathrm{~h}$ in the OA group compared with the control group; however, this was rapidly downregulated at $6 \mathrm{~h}$ (Fig. 1A). The expression levels of miR-21 (Fig. 1B) increased from an early stage of OA-induced ALI and gradually increased such that the expression levels showed a maximum at $24 \mathrm{~h}$, whereas the downregulation of miR-1 (Fig. 1C) was significantly different between the groups at $24 \mathrm{~h}$. No differences in the changes of the expression ratio of miR-132 were observed between the groups.

Target prediction and bioinformatics analysis. Differentially expressed miRNAs were put through target gene prediction. Since each miRNA potentially regulates a large number of targets, two miRNA target databases were used to improve the accuracy of target prediction. The DAVID tool was employed to further clarify the biological significance of these potential targets and the results provided several functional biological themes.

miR-101a yielded 199 possible gene targets with enrichment scores $>0.7$ in three functional categories. The most significant targets were phosphoprotein, transferase and serine/threonine protein kinase. miRNA-1 yielded targets in a functional cluster with the most enriched targets, including vesicle, cytoplasmic vesicle, membrane-bounded vesicle and cytoplasmic membrane-bounded vesicle with an enrichment score of 5.11. miRNA-21 targeted 51 cluster genes. The highest annotation clustering with an enrichment score of 2.14 was the regulation of transcription, positive regulation of macromolecule biosynthesis and transcriptional activity.

Western blot analysis of PTEN, Akt, JNK and ERK. To examine the expression levels of PTEN and Akt as targets of miR-21, western blot analysis was performed for the OA-induced ALI lung tissue of rats. At $3 \mathrm{~h}$ after OA-induced ALI, the intensity of the PTEN band decreased in the OA group in comparison with the control group and that of the p-Akt/Akt was increased. At $6 \mathrm{~h}$, the intensity of PTEN was slightly increased and p-Akt/Akt was decreased to levels similar to basal levels such that no differences between the groups were identified. Additionally, no differences were noted from 6 to $24 \mathrm{~h}$.

Serine/threonine protein kinase activity was evaluated as a putative target of miR-101 by western blot analysis. From 3 to $6 \mathrm{~h}$, the ratio of $\mathrm{p}-\mathrm{JNK} / \mathrm{JNK}$ was significantly higher in the OA group than in the control group, whereas the restored expression was observed in the OA group following $6 \mathrm{~h}$ with small differences in the control group. However, the ratio of p-ERK/ERK was not any different between the two groups over time (Fig. 3).

Immunohistochemical staining of $\alpha-S M A$. Immunohistochemistry was used to assess the expression of $\alpha$-SMA in the bronchial and alveolar wall following 6 and $24 \mathrm{~h}$ after OA-induced ALI in rats. As shown in Fig. 4, immunohistochemical staining for $\alpha$-SMA was increased in alveolar septa from $6 \mathrm{~h}$ and peaked at $24 \mathrm{~h}$. Increased diaminobenzidine staining on the smooth muscle of vessels and bronchioles was observed in the OA group. Alveolar wall edema and 
Table I. Quantitative polymerase chain reaction analysis of selected miRNAs at 3, 6 and $24 \mathrm{~h}$ after OA-induced ALI in rats.

\begin{tabular}{|c|c|c|c|c|c|c|c|c|c|}
\hline \multirow[b]{2}{*}{ Regulation } & \multicolumn{3}{|c|}{$3 \mathrm{~h}$ after OA-induced ALI } & \multicolumn{3}{|c|}{$6 \mathrm{~h}$ after $\mathrm{OA}$-induced ALI } & \multicolumn{3}{|c|}{$24 \mathrm{~h}$ after OA-induced ALI } \\
\hline & miRNA & $\mathrm{FC}$ & P-value & miRNA & $\mathrm{FC}$ & $\mathrm{P}$-value & miRNA & $\mathrm{FC}$ & P-value \\
\hline \multirow[t]{35}{*}{ Up } & rno-miR-764 & 2.85 & 0.27 & rno-miR-465 & 3.67 & 0.09 & rno-miR-21 & 3.59 & 0.01 \\
\hline & rno-miR-101a & 2.77 & 0.02 & rno-miR-21 & 3.61 & 0.02 & rno-miR-184 & 3.49 & 0.15 \\
\hline & rno-miR-297 & 2.63 & 0.23 & rno-miR-122 & 3.59 & 0.16 & rno-miR-541 & 3.26 & 0.03 \\
\hline & rno-miR-122 & 2.43 & 0.15 & rno-miR-212 & 3.37 & 0.04 & rno-miR-212 & 3.22 & 0.27 \\
\hline & rno-miR-291a & 2.34 & 0.34 & rno-miR-764 & 2.85 & 0.30 & rno-miR-132 & 3.02 & 0.06 \\
\hline & rno-miR-132 & 2.20 & 0.04 & rno-miR-132 & 2.67 & 0.02 & rno-let-7e & 2.73 & 0.02 \\
\hline & rno-miR-598 & 1.90 & 0.12 & rno-miR-297 & 2.63 & 0.25 & rno-let-7i & 2.70 & 0.34 \\
\hline & rno-miR-212 & 1.86 & 0.06 & rno-miR-291a & 2.34 & 0.37 & rno-miR-188 & 2.21 & 0.05 \\
\hline & rno-miR-298 & 1.84 & 0.20 & rno-miR-223 & 1.93 & 0.02 & rno-miR-122 & 2.17 & 0.12 \\
\hline & rno-miR-147 & 1.82 & 0.01 & rno-miR-598 & 1.90 & 0.13 & rno-miR-134 & 2.11 & 0.03 \\
\hline & rno-miR-466b & 1.76 & 0.25 & rno-miR-298 & 1.84 & 0.22 & rno-miR-382 & 2.08 & 0.20 \\
\hline & rno-let-7i & 1.73 & 0.19 & rno-miR-147 & 1.82 & 0.01 & rno-miR-146b & 1.99 & 0.01 \\
\hline & rno-miR-296 & 1.71 & 0.12 & rno-miR-466b & 1.76 & 0.27 & rno-miR-127 & 1.98 & 0.25 \\
\hline & rno-miR-493 & 1.70 & 0.39 & rno-miR-296 & 1.71 & 0.13 & rno-miR-186 & 1.96 & 0.12 \\
\hline & rno-miR-465 & 1.66 & 0.08 & rno-miR-493 & 1.70 & 0.43 & rno-miR-298 & 1.95 & 0.39 \\
\hline & rno-miR-383 & 1.65 & 0.30 & rno-miR-383 & 1.65 & 0.32 & rno-miR-376a & 1.94 & 0.12 \\
\hline & rno-miR-223 & 1.63 & 0.68 & rno-miR-333 & 1.62 & 0.10 & rno-miR-370 & 1.80 & 0.03 \\
\hline & rno-miR-333 & 1.62 & 0.09 & rno-miR-184 & 1.61 & 0.27 & rno-miR-598 & 1.76 & 0.08 \\
\hline & rno-miR-184 & 1.61 & 0.25 & rno-miR-483 & 1.55 & 0.08 & rno-miR-147 & 1.76 & 0.16 \\
\hline & rno-miR-483 & 1.55 & 0.07 & rno-miR-146b & 1.54 & 0.04 & rno-miR-324 & 1.75 & 0.21 \\
\hline & rno-miR-146b & 1.54 & 0.03 & rno-let-7i & 1.52 & 0.21 & rno-miR-483 & 1.72 & 0.02 \\
\hline & rno-miR-21 & 1.53 & 0.01 & & & & rno-miR-296 & 1.71 & 0.50 \\
\hline & & & & & & & rno-miR-431 & 1.69 & 0.34 \\
\hline & & & & & & & rno-miR-20b & 1.68 & 0.33 \\
\hline & & & & & & & rno-miR-300 & 1.67 & 0.34 \\
\hline & & & & & & & rno-miR-106b & 1.62 & 0.00 \\
\hline & & & & & & & rno-miR-494 & 1.61 & 0.25 \\
\hline & & & & & & & rno-miR-193 & 1.61 & 0.35 \\
\hline & & & & & & & rno-miR-31 & 1.60 & 0.00 \\
\hline & & & & & & & rno-miR-205 & 1.60 & 0.27 \\
\hline & & & & & & & rno-miR-678 & 1.58 & 0.32 \\
\hline & & & & & & & rno-miR-29c & 1.57 & 0.10 \\
\hline & & & & & & & rno-miR-433 & 1.55 & 0.06 \\
\hline & & & & & & & rno-miR-223 & 1.54 & 0.75 \\
\hline & & & & & & & rno-miR-465 & 1.50 & 0.40 \\
\hline \multirow[t]{15}{*}{ Down } & rno-miR-218 & 1.89 & 0.06 & rno-miR-101a & 1.90 & 0.05 & rno-miR-1 & 5.49 & 0.04 \\
\hline & rno-miR-542 & 1.87 & 0.03 & rno-miR-218 & 1.89 & 0.06 & rno-miR-218 & 2.52 & 0.02 \\
\hline & rno-miR-450a & 1.67 & 0.12 & rno-miR-542 & 1.87 & 0.03 & rno-miR-451 & 2.14 & 0.06 \\
\hline & rno-miR-374 & 1.66 & 0.03 & rno-miR-335 & 1.75 & 0.04 & rno-miR-374 & 1.98 & 0.04 \\
\hline & & & & rno-miR-450a & 1.67 & 0.13 & rno-miR-335 & 1.89 & 0.03 \\
\hline & & & & rno-miR-374 & 1.66 & 0.03 & rno-miR-542 & 1.87 & 0.08 \\
\hline & & & & rno-miR-451 & 1.65 & 0.08 & rno-miR-489 & 1.87 & 0.30 \\
\hline & & & & rno-miR-1 & 1.47 & 0.42 & rno-miR-133b & 1.81 & 0.25 \\
\hline & & & & & & & rno-miR-196c & 1.81 & 0.07 \\
\hline & & & & & & & rno-miR-206 & 1.78 & 0.39 \\
\hline & & & & & & & rno-miR-582 & 1.78 & 0.36 \\
\hline & & & & & & & rno-miR-455 & 1.76 & 0.30 \\
\hline & & & & & & & rno-miR-743a & 1.73 & 0.00 \\
\hline & & & & & & & rno-miR-24-1 & 1.71 & 0.19 \\
\hline & & & & & & & rno-miR-881 & 1.70 & 0.34 \\
\hline
\end{tabular}


Table I. Continued.

\begin{tabular}{|c|c|c|c|c|c|c|c|c|c|}
\hline \multirow[b]{2}{*}{ Regulation } & \multicolumn{3}{|c|}{$3 \mathrm{~h}$ after $\mathrm{OA}$-induced ALI } & \multicolumn{3}{|c|}{$6 \mathrm{~h}$ after $\mathrm{OA}$-induced ALI } & \multicolumn{3}{|c|}{$24 \mathrm{~h}$ after $\mathrm{OA}$-induced ALI } \\
\hline & miRNA & $\mathrm{FC}$ & P-value & miRNA & $\mathrm{FC}$ & P-value & miRNA & $\mathrm{FC}$ & P-value \\
\hline & & & & & & & rno-miR-880 & 1.67 & 0.60 \\
\hline & & & & & & & rno-miR-142 & 1.67 & 0.15 \\
\hline & & & & & & & rno-miR-33 & 1.67 & 0.10 \\
\hline & & & & & & & rno-miR-592 & 1.66 & 0.30 \\
\hline & & & & & & & rno-miR-101a & 1.66 & 0.12 \\
\hline & & & & & & & rno-miR-192 & 1.65 & 0.04 \\
\hline & & & & & & & rno-miR-7a & 1.65 & 0.02 \\
\hline & & & & & & & rno-miR-323 & 1.63 & 0.08 \\
\hline & & & & & & & rno-miR-10a & 1.62 & 0.30 \\
\hline & & & & & & & rno-miR-96 & 1.57 & 0.90 \\
\hline & & & & & & & rno-miR-471 & 1.56 & 0.05 \\
\hline & & & & & & & rno-miR-194 & 1.54 & 0.35 \\
\hline & & & & & & & rno-miR-382 & 1.54 & 0.15 \\
\hline & & & & & & & rno-miR-19a & 1.52 & 0.01 \\
\hline
\end{tabular}

OA, oleic acid; ALI, acute lung injury; miRNA, microRNA; FC, fold change.
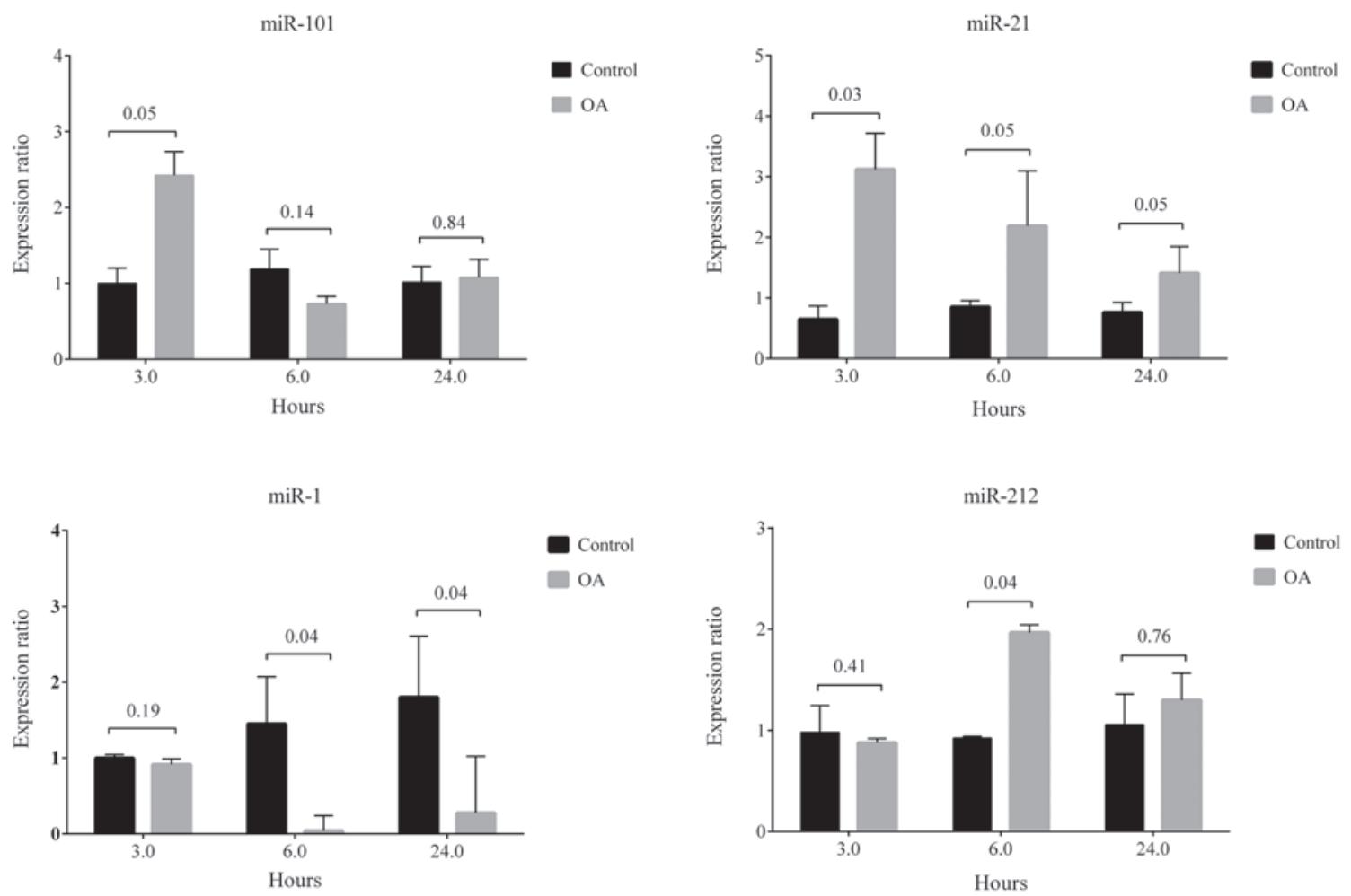

Figure 1. Quantitative polymerase chain reaction with primers specific to the miRNA of interest 3,6 and $24 \mathrm{~h}$ after OA-induced ALI. The relative expression of each mature miRNA was evaluated using the $\mathrm{C}_{\mathrm{T}}$ method and was normalized to those of the internal control miR-202 for each condition $\left(\Delta \Delta \mathrm{C}_{\mathrm{T}}\right)$. Quantitative data representing the relative expression ratio of a target gene was calculated versus the control. The mean \pm standard error from three experiments performed in duplicates are presented in the bar graphs. P-values were calculated using the Mann-Whitney U-test or unpaired t-test between the control group and the OA group. miRNA, microRNA; OA; oleic acid; ALI, acute lung injury; $\mathrm{C}_{\mathrm{T}}$, comparative threshold cycle.

mucus secretion in the alveolar space were observed following $6 \mathrm{~h}$ and these were notably increased $24 \mathrm{~h}$ after OA-induced ALI.
IL-13 concentration in the BAL supernatant. The IL- 3 concentration in the BAL fluid did not differ between the control and OA-induced ALI rats from 3 to $24 \mathrm{~h}$ after challenge (Fig. 3). 


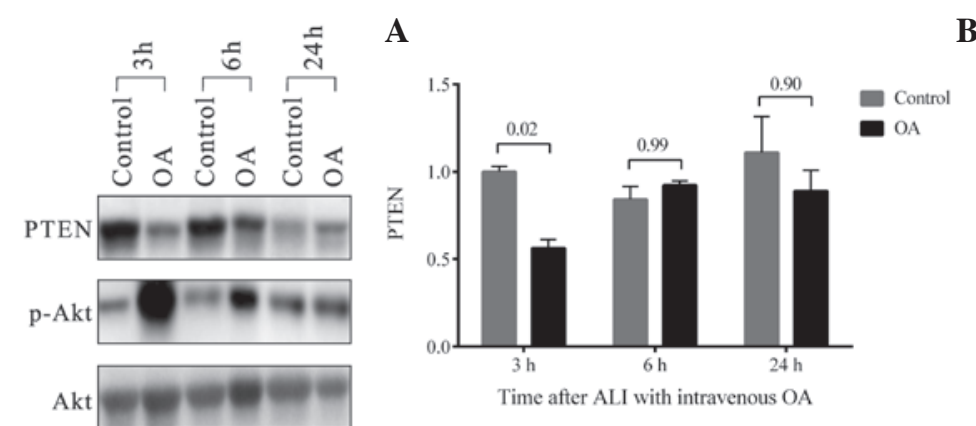

$\mathbf{B}$

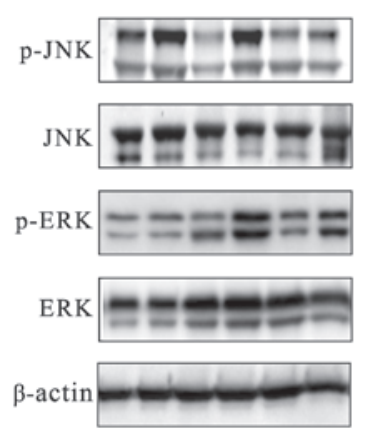

C

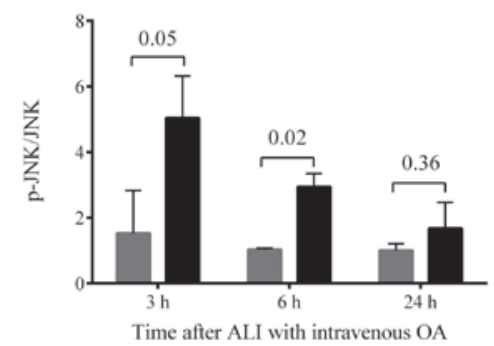

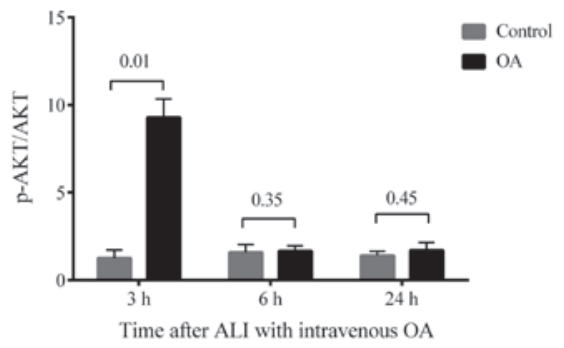

D

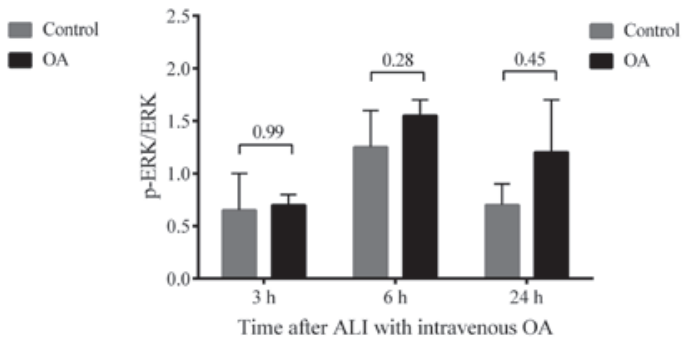

Figure 2. Western blot analysis of lung tissue was performed 3, 6 and $24 \mathrm{~h}$ after OA-induced ALI with antibodies against PTEN, Akt, JNK and ERK. PTEN inhibition increases Akt and JNK signaling. (A) P-PTEN/PTEN was increased at $3 \mathrm{~h}$ in the OA group compared with the control. (B) P-Akt/Akt was significantly increased in the OA group at $3 \mathrm{~h}$ and subsequently decreased to basal levels $6 \mathrm{~h}$ after OA-induced ALI. (C) P-JNK/JNK activity mildly increased at $3 \mathrm{~h}$ and substantially increased until $6 \mathrm{~h}$. (D) P-ERK/ERK ratio was not altered following the OA challenge. P-values were calculated from the Mann-Whitney U-test or unpaired t-test between the control and OA group. ALI, acute lung injury; OA, oleic acid; PTEN, phosphatase and tensin homolog; p-Akt, phosphorylated protein kinase B; JNK, c-Jun N-terminal kinase; ERK, extracellular signal-regulated kinases.

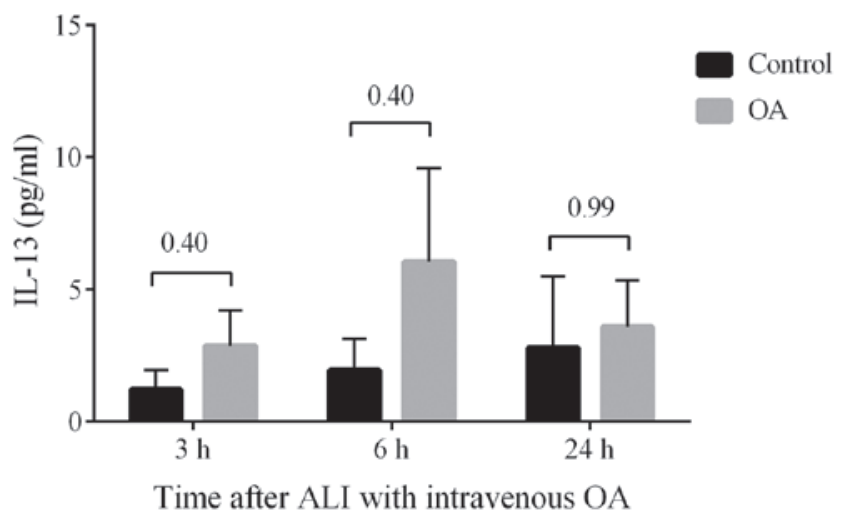

Figure 3. IL-13 levels in BAL fluid were measured using ELISA. The concentration of IL-13 was compared between the groups using the Mann-Whitney $\mathrm{U}$ test. IL-13 concentration was increased at $6 \mathrm{~h}$ for the OA group; however, this difference was not statistically significant. BAL, bronchoalveolar lavage; OA, oleic acid.

\section{Discussion}

The objective of the present study was to examine the expression profile of miRNAs following ALI induced by intravenous administration of OA and to identify miRNAs potentially mediating possible mechanisms of OA-induced ALI. Several miRNAs demonstrated distinctive characteristic expressions with different temporal patterns. In the early phase of OA-induced ALI, miR-101a expression was significantly increased and a superimposed increase in the $\mathrm{p}-\mathrm{JNK} / \mathrm{JNK}$ ratio was observed. Subsequently, the expression levels of miR-101a rapidly decreased following $6 \mathrm{~h}$. miR-21 was upregulated from 3 to $24 \mathrm{~h}$; however, miR-1 was downregulated more slowly by $24 \mathrm{~h}$. miR-21 is known to target the 3'-untranslated region (UTR) of PTEN mRNA to decrease PTEN activity and to augment Akt phosphorylation in lung tissue. $\alpha$-SMA expression in the lung tissue as a target of miR-1 and miR-21 was also increased at late phases of OA-induced ALI.

The results of the present study endorse the hypothesis that microRNAs mediate OA-induced lung injury. Transferase and serine/threonine protein kinase were selected as putative target genes of miR-101, which was the most rapidly upregulated microRNA. The present study further investigated the expression of mitogen-activated protein kinase (MAPK) in OA-induced ALI for the target miR-101. ERK1/2, the most extensively studied group of mammalian MAPKs, are considered to regulate the initiation and progression of LPS-mediated neutrophil accumulation and inflammation in the lung and consequently mediate ALI (11). Gonçalves-de-Albuquerque et al (12) reported that OA-induced ALI is dependent on ERK1/2 activation and that the inhibition of ERK phosphorylation attenuates lung injury in mice. However, another study reported that OA affects the expression of p38 and JNK in the early phase of ALI response to variant stimulation, including proinflammatory cytokines (13). MAPK phosphatase-1 (MKP-1) is a typical member of the MKP family that is essential in the feedback control of p38 and JNK; however, it acts as a weak inhibitor of ERK phosphorylation. Zhu et al (14) reported that the 3'-UTR of MKP-1 mRNA has a conserved complementary site for the complementary region of miR-101, which directly suppresses MKP-1, and transfection of miR-101 attenuates the activity of MKP-1 and eventually induces the activation of JNK and p38, but not ERK in cultured cell lines (14). 


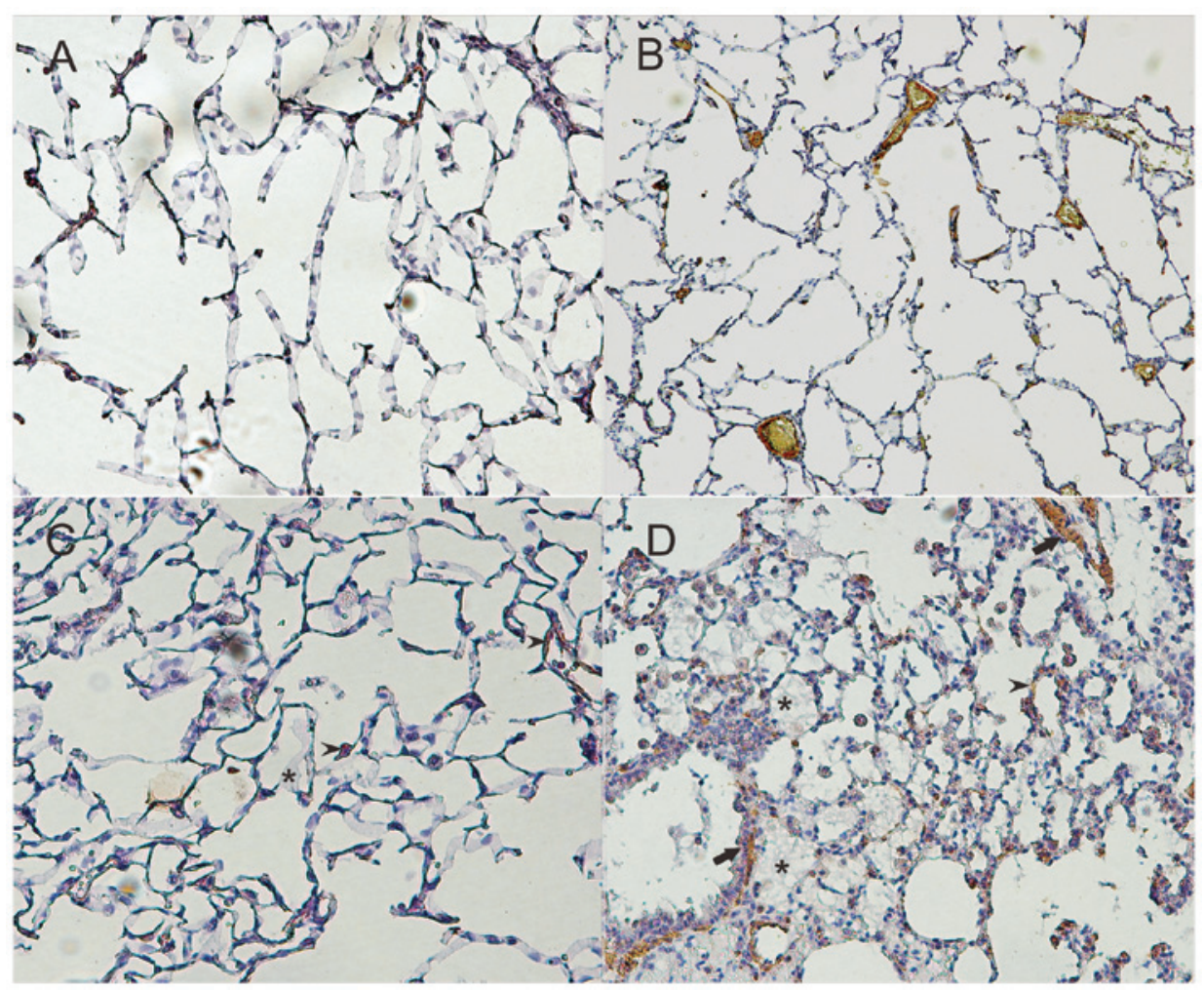

Figure 4. IHC with monoclonal anti- $\alpha$-actin antibody was performed 6 and $24 \mathrm{~h}$ after OA-induced ALI. The figures are representative microphotographs from each category. (A) IHC of a control group sample at $6 \mathrm{~h}$. Positivity for $\alpha$-SMA is observed in tangentially sectioned basal epithelial cells and along the BM of bronchial epithelium; (B) IHC of the control group $24 \mathrm{~h}$ after OA-induced ALI. Fluorescent activity in the alveolar wall was similar to the IHC result at $6 \mathrm{~h}$; (C) IHC of the OA group sample $6 \mathrm{~h}$ after OA-induced ALI. Alveolar wall edema and mucus secretion in the alveolar space were observed (asterisk). DAB staining in the epithelial cells (arrowhead) and in a wide area of stromal connective tissue underneath the BM was observed. (D) Increased DAB staining in the alveolar BM (arrowhead), smooth muscle of the bronchial wall or of the blood vessels (arrows) was revealed $24 \mathrm{~h}$ after OA-induced ALI. Thick mucus secretion was found in the alveolar spaces (asterisk) $24 \mathrm{~h}$ after OA-induced ALI. IHC, immunohistochemical staining; OA, oleic acid; ALI, acute lung injury; $\mathrm{BM}$, basement membrane; DAB, diaminobenzidine.

Lager et al (15) have suggested that OA modulates the toll-like receptor 4 (TLR4) signaling pathway and that activation of TLR4 results in the transcription of JNK and p38 MAPK. In a similar study using a murine alveolar macrophage cell line, Zhu et al (14) reported that several TLR ligands increased miR-101 expression levels in a time-dependent manner, with a peak at 90 min following stimulation. Despite the fact that this study was performed on an in vivo model, increased levels of miR-101a expression were also observed in the early phase of OA-induced ALI, which was followed by increased JNK phosphorylation. However, the present study did not assess how miR-101 mediates the TLR ligand to JNK and p38 MAPK following OA injection. Further study is required to evaluate the association between miR-101 and the TLR signaling pathway in ALI.

The present study also demonstrated upregulated miR-21 expression $24 \mathrm{~h}$ following OA-induced ALI and downregulation of miR-1 in the late phase of OA-induced ALI. miR-21 is a miRNA which is highly conserved among vertebrates and it is known to have vital functions in immunological reactions. A study of miRNA expression profiling during ALI from aerosolized LPS in an animal model demonstrated that miR-21 performs a pivotal function in inflammatory responses (7). Additionally, the expression of miR-21 is significantly induced in alveolar epithelial cells and infiltrating macrophages in hyperoxia-induced ALI, whereas knocking down miR-21 using antisense probes attenuated pulmonary fibrosis in mice treated with bleomycin $(16,17)$. In the present study, miR-21 was the most upregulated miRNA following IV OA administration and maintained steady expression levels throughout the progression of OA-induced ALI.

PTEN is a multifunctional phosphatase that negatively regulates the phosphoinositide 3-kinase (PI3K)/Akt pathway, which has a regulatory role in lung fibroblasts and subsequent lung scarring. A previous study has reported that a reduction in PTEN expression and hyperactivation of Akt are associated with pulmonary fibrosis (18). Previous studies have demonstrated that miR-21 is able to regulate cell growth and survival by binding to the 3'-UTR of PTEN mRNA, which contains a miR-21 recognizing element (19). The increased PTEN expression activity by the downregulation of miR-21 was able to be reversed by the transfection of miR-21, indicating a critical regulation of PTEN by miR-21 (16). The present study also detected inactivation of PTEN by OA and an inverse association between miR-21 and the expression of PTEN.

In the present study, total PTEN expression was attenuated and the expression of p-Akt was significantly increased $3 \mathrm{~h}$ after OA-induced ALI. As the final outcome, OA repressed PTEN expression and activated p-Akt through increasing miRNA-21 expression. However, a previous study reported that a PTEN inhibitor, which stimulates p-Akt activity and p-ERK expression, attenuated lung injury. Therefore, 
Akt/PI3K signaling has beneficial effects in ALI (20). In another study, mice were subjected to postnatal targeted deletion of the PTEN gene with the (tetO) $)_{7}$-Cre system in the lung epithelium. The authors demonstrated that OA induces mTOR-dependent miR-21 upregulation and miR-21 specifically targets PTEN mRNA resulting in repressed expression of PTEN, increased expression of Akt and finally resulted in exacerbated ALI (18).

Thum et al (21) reported that miR-21 induces the ERK signaling pathway through the inhibition of sprout homologue 1 in cardiac fibroblasts. However, this study did not reveal ERK activation in OA-induced ALI despite significant expression changes in miR-21, which may be associated with differences in organs and thus requires further study.

Lamers et al (22) reported that either OA alone or OA in combination with adipokines is able to induce the proliferation and migration of human vascular smooth muscle cells. Vascular smooth muscle cells are highly specialized cells that are important in the control of blood vessel changes, including atherosclerosis. Another study has demonstrated that muscle-specific miR-1 is a key regulator of cardiac and skeletal muscle hypertrophy and it has a linear inverse correlation with pathological conditions (23). Myofibroblasts, which are activated from fibroblasts, express $\alpha$-SMA as a marker of fibroblast activation, and its presence indicates the differentiation of fibroblasts into myofibroblasts. A previous study reported that miR-1 regulates smooth muscle cell differentiation and that silencing miR-1 enhances the expression of smooth muscle-restricted contractile proteins (24). These studies have highlighted that a constant expression of miR-1 is important for normal muscular physiology and that the downregulation of $\mathrm{miR}-1$ results in smooth muscle hypertrophy. The present study demonstrated that miR-1 is the most downregulated miRNA and that it increased $\alpha$-SMA expression in OA-induced ALI. miR-21 is known to upregulate smooth-muscle contractile proteins by silencing programmed cell death 4 (25). Additionally, Ohtani et al demonstrated that, through the control of PTEN, miR-21 has a significant role in the differentiation, proliferation and survival of vascular smooth muscle cells (26). The upregulation of miR-21 in conjunction with the downregulation of miR-1 has a critical function in OA-induced ALI in rats.

Lu et al (27) identified an expression profile of miRNAs similar to that in the present study, by upregulation of miR-21 and downregulation of miR-1 expression in three different types of allergic airway inflammation models. It may be postulated that OA-induced ALI is mediated by allergic airway inflammation in the way that upregulation of miR-21 and downregulation of miR-1 were observed over the whole period of OA-induced ALI. In a previous study, anti-allergic drug treatment was demonstrated to prevent increases in pulmonary vascular permeability and the decrease in arterial partial oxygen pressure, which was induced by IV OA (28). However, the results of the present study did not identify increment changes in IL-13 levels in BAL fluid, which is a potent target of miR-21 and the most important cytokine in allergic airway inflammation triggering macrophage and eosinophil activation, immunoglobulin E production and smooth muscle cell activation. Thus, it cannot be postulated that the allergic reaction mediates $\mathrm{OA}$-induced $\mathrm{ALI}$.
The present study assessed changes in miRNA levels and simultaneous alteration with the expression of inflammatory mediators. However, changes in inflammation induced by IV OA following the inhibition or transfection of differentially expressed miRNAs were not investigated, such that a transfection study with siRNA is required to definitize the biological mechanisms.

In conclusion, the present study revealed that several miRNAs are involved in the regulation of OA-induced ALI with dynamic changes in temporal patterns. miR-101a levels were increased in the early phase of OA-induced ALI, which is associated with MKP-1 activation and JNK phosphorylation, which, in turn, mediates the cellular response to various stimuli, including infection and environmental factors. The upregulation of miR-21 and downregulation of miR-1 appeared in the early and late phases of OA-induced ALI. The inactivation of PTEN and activation of the Akt pathway were observed simultaneously. miR-21 directly targets PTEN and miR-1 stimulates airway smooth muscle cell proliferation.

\section{Acknowledgements}

This study was supported by The Catholic University of Korea Daejeon St. Mary's Hospital, Clinical Research Institute Grant funded by The Catholic University of Korea Daejeon St. Mary's Hospital (no. CMCDJ-A-2012-006). This study was also supported by Daejeon Health Sciences College in 2012.

\section{References}

1. Bernard GR: Acute respiratory distress syndrome: a historical perspective. Am J Respir Crit Care Med 172: 798-806, 2005.

2. Schuster DP: ARDS: clinical lessons from the oleic acid model of acute lung injury. Am J Respir Crit Care Med 149: 245-260, 1994.

3. Derks CM and Jacobovitz-Derks D: Embolic pneumopathy induced by oleic acid. A systematic morphologic study. Am J Pathol 87: 143-158, 1977

4. Dickey BF, Thrall RS, McCormick JR and Ward PA: Oleic-acid-induced lung injury in the rat. Failure of indomethacin treatment or complement depletion to ablate lung injury. Am J Pathol 103: 376-383, 1981.

5. Ambros V: The functions of animal microRNAs. Nature 431: 350-355, 2004.

6. Zhou T, Garcia JG and Zhang W: Integrating microRNAs into a system biology approach to acute lung injury. Transl Res 157: 180-190, 2011.

7. Moschos SA, Williams AE, Perry MM, Birrell MA, Belvisi MG and Lindsay MA: Expression profiling in vivo demonstrates rapid changes in lung microRNA levels following lipopolysaccharide-induced inflammation but not in the anti-inflammatory action of glucocorticoids. BMC Genomics 8: 240, 2007.

8. Xie T, Liang J, Guo R, Liu N, Noble PW and Jiang D: Comprehensive microRNA analysis in bleomycin-induced pulmonary fibrosis identifies multiple sites of molecular regulation. Physiol Genomics 43: 479-487, 2011.

9. Wang X: miRDB: A microRNA target prediction and functional annotation database with a wiki interface. RNA 14: 1012-1017, 2008.

10. Lewis BP, Burge CB and Bartel DP: Conserved seed pairing, often flanked by adenosines, indicates that thousands of human genes are microRNA targets. Cell 120: 15-20, 2005.

11. Fang WF, Cho JH, He Q, et al: Lipid A fraction of LPS induces a discrete MAPK activation in acute lung injury. Am J Physiol Lung Cell Mol Physiol 293: L336-L344, 2007.

12. Gonçalves-de-Albuquerque CF, Silva AR, Burth P, et al: Oleic acid induces lung injury in mice through activation of the ERK pathway. Mediators Inflamm 2012: 956509, 2012. 
13. Nick JA, Young SK, Brown KK, et al: Role of p38 mitogen-activated protein kinase in a murine model of pulmonary inflammation. J Immunol 164: 2151-2159, 2000.

14. Zhu QY, Liu Q, Chen JX, Lan K and Ge BX: MicroRNA-101 targets MAPK phosphatase-1 to regulate the activation of MAPKs in macrophages. J Immunol 185: 7435-7442, 2010.

15. Lager S, Gaccioli F, Ramirez VI, Jones HN, Jansson T and Powell TL: Oleic acid stimulates system A amino acid transport in primary human trophoblast cells mediated by toll-like receptor 4. J Lipid Res 54: 725-733, 2013.

16. Liu G, Friggeri A, Yang Y, et al: miR-21 mediates fibrogenic activation of pulmonary fibroblasts and lung fibrosis. J Exp Med 207: $1589-1597,2010$

17. Bhaskaran M, Xi D, Wang Y, et al: Identification of microRNAs changed in the neonatal lungs in response to hyperoxia exposure. Physiol Genomics 44: 970-980, 2012.

18. Miyoshi K, Yanagi S, Kawahara K, et al: Epithelial Pten controls acute lung injury and fibrosis by regulating alveolar epithelial cell integrity. Am J Respir Crit Care Med 187: 262-275, 2013.

19. Qin X, Yan L, Zhao X, Li C and Fu Y: microRNA-21 overexpression contributes to cell proliferation by targeting PTEN in endometrioid endometrial cancer. Oncol Lett 4: 1290-1296, 2012.

20. Lai JP, Bao S, Davis IC and Knoell DL: Inhibition of the phosphatase PTEN protects mice against oleic acid-induced acute lung injury. Br J Pharmacol 156: 189-200, 2009.
21. Thum T, Gross C, Fiedler J, et al: MicroRNA-21 contributes to myocardial disease by stimulating MAP kinase signalling in fibroblasts. Nature 456: 980-984, 2008.

22. Lamers D, Schlich R, Greulich S, Sasson S, Sell H and Eckel J: Oleic acid and adipokines synergize in inducing proliferation and inflammatory signalling in human vascular smooth muscle cells. J Cell Mol Med 15: 1177-1188, 2011.

23. Elia L, Contu R, Quintavalle M, et al: Reciprocal regulation of microRNA-1 and insulin-like growth factor-1 signal transduction cascade in cardiac and skeletal muscle in physiological and pathological conditions. Circulation 120: 2377-2385, 2009.

24. Xie C, Huang H, Sun X, et al: MicroRNA-1 regulates smooth muscle cell differentiation by repressing Kruppel-like factor 4. Stem Cells Dev 20: 205-210, 2011.

25. Kang $\mathrm{H}$ and Hata A: MicroRNA regulation of smooth muscle gene expression and phenotype. Curr Opin Hematol 19: 224-231, 2012.

26. Ohtani K and Dimmeler S: Contol of cardiovascular differentiation by microRNAs. Basic Res Cardiol 106: 5-11, 2011.

27. Lu TX, Munitz A and Rothenberg ME: MicroRNA-21 is up-regulated in allergic airway inflammation and regulates IL-12p35 expression. J Immunol 182: 4994-5002, 2009.

28. Ishitsuka Y, Moriuchi H, Yang C, et al: Preventive effect of tranilast on oleic acid-induced lung injury in guinea pigs. Biol Pharm Bull 27: 1451-1454, 2004. 